\title{
Acute Cough and Urinary Leakage in Adult Women
}

\section{Alexander $K^{*}$, Tzipi S, Amnon L and Reuven D}

Clalit Health Center, Hebrew University, Jerusalem, Israel

\begin{abstract}
Background: Upper respiratory tract infection (URTI) is the most frequent reason for patients' visits in primary care. We assessed the proportion of adult women suffering from URTI that reported cough-induced urinary leakage.

Methods: We performed prospective field prevalence study, during it we surveyed consecutive women suffering from URTI and cough concerning their urinary symptoms.

Results: We surveyed 208 women, age 31-88 years (mean 54.8 years). Fifty three per cent of women that visited their FPs with URTI, reported urinary leakage as one of their complaints. The majority of them never experienced involuntary urinary leakage. $25 \%$ of patients with urinary leakage considered this symptom as "serious" or "very serious". Women that experienced urinary leakage with URTI almost never complained about urinary leakage to their caregivers and were almost never questioned about the presence of urinary leakage during a visit for URTI. Nevertheless, urinary leakage can be very troublesome, but potentially treatable.
\end{abstract}

Conclusion: Urinary leakage is frequent troublesome symptom of URTI, yet it is largely underreported. Primary care physicians rarely question as to the presence of urinary leakage during URTI. The physician should ask actively about urinary leakage.

Keywords: Cough; Urinary leakage; Aged people

\section{Introduction}

Involuntary leakage of urine is a frequent complaint among women. Attempts to assess its prevalence have brought a variety of diverse results [1-4]. According to subjective cultural norms, women can live with urinary leakage as an unpleasant but otherwise normal occurrence, such as menstruation or sweating. Otherwise, women may be willing, under certain circumstances, to discuss this issue and even be eager to hear suggestions as to its management. Women suffering from urinary leakage may even report the issue to their treating physician, if physician rapport is adequate and if they consider their problem as deserving attention. When asked, many women reported in previous studies that their urinary incontinence is frequently related to cough $[3,4]$.

Acute upper respiratory infection (herein Acute URI) is the most frequent reason for patient visits in primary care. This is usually a mild and self-limited disease. The definition of acute URI is usually a diagnosis of exclusion. The treatment of acute URI classically is focused upon ameliorating its associated symptoms. We search therefore to answer to several questions:

1. What is the prevalence of urinary leakage in adult women suffering from acute URI?

2. How many of these women consider their urinary leakage to be significant?

3. Do physicians ask the relevant questions regarding the presence of urinary leakage associated with acute URI?

4. Are age, ethnicity and religious status predictive of physicians asking about the presence of urinary leakage associated with acute URI?

\section{Materials and Methods}

Our study was performed during usual clinical visits in three family practices in Jerusalem area.

The population of those clinics consists of low- to middle-income families. Patients' medical files are computerized and visits are recorded in the medical file according to these template headings: complaints, findings, diagnosis, referrals, treatment and sick leave.

We performed prospective field prevalence study between October 2012 and April 2014. During the visit in which the doctor diagnosed Acute URI with cough (as a major complaint), women above 30 years or age were asked several questions regarding cough-related urinary leakage. These answers and other demographic data were collected. Women with known diagnosis of stress incontinence, women that suffered from cough-related chronic diseases, pregnant women and women that could not understand doctor's questions were excluded from the study.

\section{The study questions were}

Is the cough in your current disease associated with involuntary wetting?

If yes, how does this wetting affect your quality of life (does not, easily, moderately, severely, very severely)?

Did you suffer in past from events of acute cough-related wetting?

If yes, did you report this complaint to your physician or other medical professional?

Did your physician ever asked you about involuntary wetting during acute disease?

If you suffered in past from events of acute cough-related wetting, did you receive any treatment?

*Corresponding author: Alexander Kiderman, Clalit Health Center, Hebrew University, Jerusalem, Israel, Tel: 972- 02-5359111; Fax: 972-02- 5352777; E-mail: kiderman@clalit.org.il

Received September 28, 2016; Accepted December 26, 2016; Published December 30, 2016

Citation: Alexander K, Tzipi S, Amnon L, Reuven D (2016) Acute Cough and Urinary Leakage in Adult Women. Primary Health Care 6: 250. doi:10.4172/21671079.1000250

Copyright: (c) 2016 Alexander K, et al. This is an open-access article distributed under the terms of the Creative Commons Attribution License, which permits unrestricted use, distribution, and reproduction in any medium, provided the original author and source are credited. 
To achieve targets of our study, we calculated the prevalence of urinary leakage in adult women with acute URI with cough and the prevalence of women that considered this event as a significant disturbance to their quality of life. As well, we assessed the extent of medical professionals involvement in this problem and influence of several demographic variables of our patients on physicians' involvement.

\section{Statistical Methods}

We used descriptive statistics to describe the women population and investigate the frequencies of incontinent complains.

We used the t-test for univariate analysis of normal distributed variants and $\mathrm{Chi}^{2}$ for categorical variables. We used logistic regression to control for co-variants.

All data were analyzed by IBM SPSS statistics 22 package.

\section{Results}

We surveyed 208 women aged 31-88 years (mean 54.8 years) (Table 1). The average number of children born to subjects in this study was $2.92 .83 .7 \%$ of the subjects had never had a caesarean delivery. Most women defined themselves as "secular" (55.2\%), followed by "traditional" (32.3\%), "religious" (11.9\%) and "other" (1.5\%). The average BMI was $27.9+5.3$.

When questioned about urinary leakage during an acute URI with cough, $52.9 \%$ percent of the subjects responded affirmatively. Leakage was described as "mild" (42.2\%), "intermediate" (32.1\%), "serious" (17.4\%), and "very serious" (8.2\%). More than two-thirds of women $(70.6 \%)$ denied prior episodes of urinary leakage with an acute URI with cough. Notably, of those that reported that they had urinary leakage in the past, $97.0 \%$ did not report this symptom to their physician. Remarkably, $98.5 \%$ of women stated that a physician had never questioned them as to the presence of urinary leakage with an acute URI with cough.

In the $t$ test for univariate analysis, none of the correlations were statistically significant.

Using the logistic regression and after controlling for age, number of vaginal deliveries, number of $\mathrm{C}$-sections and level of religiosity only BMI was positively correlated with urine incontinence (OR for any addition 5 BMI units $=1.52$, $\mathrm{p}=0.008$ ).

\section{Discussion}

This study confirms our observation that many adult women suffer from urinary leakage during acute URI. The existing medical literature observes that the prevalence of urinary incontinence in women varies substantially according to different definitions of this troublesome symptom and different settings of studies [1-6]. In our study almost $53 \%$ of subjects reported urinary leakage during URI. Anecdotally, many subjects stated that they recognized acute URI and know that there is

\begin{tabular}{|c|c|c|}
\hline Age & Average $=55$ & Median $=54$ \\
\hline Vaginal Deliveries & Average $=2.92$ & Median=3 \\
\hline \multirow[t]{2}{*}{ Cesarean Deliveries } & Average $=0.29$ & Median $=0$ \\
\hline & $174 / 208$ (83.7\% had no cesarean) & \\
\hline \multirow[t]{5}{*}{ Level of Religious Observance (self-defined) } & (7 missing) & \\
\hline & Religious & $24 / 201(11.9 \%)$ \\
\hline & Secular & $109 / 201(55.2 \%)$ \\
\hline & Traditional & $65 / 201(32.3 \%)$ \\
\hline & Other & $3 / 201(1.5 \%)$ \\
\hline BMI & Average $=27.9$ & Median=27.7 \\
\hline \multicolumn{3}{|l|}{ Present Urinary Leakage } \\
\hline & Yes-110/208 & $52.9 \%$ \\
\hline & No $-98 / 208$ & $47.1 \%$ \\
\hline \multirow[t]{5}{*}{ If leakage, to what extent } & (1 missing) & \\
\hline & Mild - 46/109 & $42.2 \%$ \\
\hline & Intermediate $-35 / 109$ & $32.1 \%$ \\
\hline & Serious - 19/109 & $17.4 \%$ \\
\hline & Very serious - 9/109 & $8.2 \%$ \\
\hline \multirow[t]{3}{*}{ Did you suffer from leakage in the past? } & (4 missing) & \\
\hline & Yes $-60 / 204$ & $29.4 \%$ \\
\hline & No $-144 / 204$ & $70.6 \%$ \\
\hline \multicolumn{3}{|l|}{ If leakage in past, did you report to physician? } \\
\hline & Yes - 3/60 & $5.0 \%$ \\
\hline & No $-57 / 60$ & $95.0 \%$ \\
\hline \multicolumn{3}{|c|}{ If leakage in past, did you report to a different medical professional? } \\
\hline & Yes $-1 / 60$ & $1.7 \%$ \\
\hline & No $-59 / 60$ & $98.3 \%$ \\
\hline \multicolumn{3}{|c|}{ Has a physician inquired about urinary leakage in the past? } \\
\hline & Yes - 3/203 & $1.5 \%$ \\
\hline & No $-200 / 203$ & $98.5 \%$ \\
\hline \multicolumn{3}{|l|}{ If it was identified, how was it treated } \\
\hline & Medically - 1/60 & $1.7 \%$ \\
\hline & None $-60 / 61$ & $98.4 \%$ \\
\hline
\end{tabular}

Table 1: In the table there are demographic details (age, BMI, level of religious observance), number of vaginal/cesarean deliveries, presence of urinary leakage, its' extent, urinary leakage in the past, how it was treated. 
not effective medical therapy for it. It would seem that they visited the clinic only because the urinary incontinence had become unbearable or embarrassing. More than quarter of women reported that their urinary leakage was "serious" or "very serious". Interestingly, only one of the subjects had previously complained about urinary leakage. We believe that one reason for that under- diagnosis and under- report of urinary leakage is the misconception of women and practitioners that there is no reason to speak about it because there is no effective treatment anyway. The vast majority of symptomatic women denied prior episodes of urinary incontinence. As with any self-reported information, it may be truth and may be recall bias (selective forgetfulness). We feel that if a subject reported to their physician about new symptom, it should be clear that she expected some sort medical treatment or guidance.

In this study we asked subjects that experienced previous acute URIrelated urinary incontinence about their conversations with medical providers regarding this symptom. We were surprised that nearly all of them had never complained about their leakage to a physician or nurse. They almost never were asked by their doctors about urinary leakage during URI with cough. Only one of our patients received any medical treatment during a previous symptomatic episode of incontinence.

The results of our study are no more representative than other reported results: $40 \%$ of affected women in Canada (4) or 3\% in Japan (3), but we surmise that results are culturally dependent.

We did not find significant correlations between age, number of vaginal deliveries and religiosity with reported leakage, contrary to common knowledge, but this non-finding may be explained by our small sample.

\section{Conclusion}

We have two practical recommendations from the results of our study. We encourage physicians to inquire directly as to the presence of urinary incontinence, especially in the presence of a medical condition, such as an acute URI, that may accentuate any baseline tendency for urinary leakage. Secondly, we recommend that once identified, practitioners should offer medical therapy (relieving of cough, for instance) and guidance, as clinically appropriate, to help alleviate bothersome symptoms.

\section{References}

1. Andersson G, Johansson JE, Garpenholt O, Nilsson K (2004) Urinary incontinence - prevalence, impact on daily living and desire for treatment: A population based study. Scand J Urol Nephrol 38: 125-130.

2. Keller SL (1999) Urinary incontinence: occurrence, knowledge and attitudes among women aged 55 and older in a rural Midwestern setting. $\mathrm{J}$ Wound Ostomy Continence Nurs 26: 30-38.

3. Ueda T, Tamaki M, Kageyama S, Yoshimura N, Yoshida O (2000) Urinary incontinence among community-dwelling people aged 40 years or older in Japan: prevalence, risk factors knowledge and self-perception. Int J Urol 7: 95-103.

4. Swanson JG, Kaczorovski J, Skelly J, Finkelsteim M (2005) Urinary incontinence: Common problem among women over 45. Can Fam Physician 51: 84-85

5. Dolan LM, Casson K, McDonald P, Ashe RG (1999) Urinary incontinence in Northern Ireland: A prevalence study. Br J Urinol Int 83: 760-766.

6. MacKay K, Hemmet L (2002) Needs assessment of women with urinary incontinence in a district health authority. BMJ 51: 801-804. 